\title{
Relationship between Quantum Physics and Maxwell's Equations in the Model of a Hydrogen Atom
}

\author{
Y. E. Khoroshavtsev
}

\section{ABSTRACT}

\begin{abstract}
An attempt to bring together two different theories - classical electrodynamics and quantum mechanics is made. On the example of a hydrogen atom the problem of the hypothetic electron fall into a nucleus by means of the energy conservation law is examined. The essence of the present approach consists in the assumption, that the energy and momentum of an electron in quantum model are proportional to corresponding electromagnetic fluxes. In order to achieve the result, the new formula of momentum flux density not using Poynting vector was proposed. It states that the momentum flux depends not only on electric and magnetic components of the field, but also on a frequency of an electromagnetic wave. As the main result, it was demonstrated that the total including annihilation energy of an electron in Bohr's atom model is equal to energy of a free electron $m c^{2}$ without any mention of Relativity. An electromagnetic field inside an atom occurs quantized for each electron orbit. An additional consequence shows that the two fundamental definitions of quantum energy $m c^{2}$ and $h \omega$ are interrelated. If $h \omega$ is admitted according to quantum physics, then $m c^{2}$ follows automatically and vice versa.
\end{abstract}

Keywords: Bohr's model, electrodynamics, momentum flux, Poynting vector, quantization.

\section{INTRODUCTION}

The classical electrodynamics and quantum physics study a lot the same physical processes but at the different scale. The boundary of their scopes of authority passes at the atomic level, and the mathematical apparatus are principally different.

Electrodynamics is the vector gauge theory determines electric and magnetic fields as the power characteristics of points of the space regarding the position of charges and currents.

Quantum mechanics is the scalar theory, which gives a possibility to calculate the state probabilities of elementary particles. The postulates of quantum physics do not follow from Maxwell's equations and vice versa.

The various authors proposed the modifications of theories [1]-[3]. The concurrent analysis of their strong and weak aspects can be read in [4]-[6].

Each theory has limits of application. Some observed electromagnetic phenomena are incompatible with Maxwell equations. These include the black body radiation, atom stability etc. [7]. Many experiments cannot be described not even approximately.

Despite that, Maxwell theory constitutes a step towards the Standard model in a particle physics. Its gauge procedures result of a field description of nature and symmetry of mathematical transformations [8]. Quantum electrodynamics (QED) as an abelian gauge theory, which resolved many
Published Online: September 20, 2021

ISSN: $2684-4451$

DOI :10.24018/ejphysics.2021.3.5.102

\section{Y.E. Khoroshavtsev *}

Faculty of Automatic Control Systems, State University of Civil Aviation, St. Petersburg, Russia.

(e-mail: khoroshavtsev@mail.ru)

*Corresponding Author problems and explained many experiments, was created, and gave a new knowledge of the nature [9]. However, the mathematical tools of QED are totally unlike Maxwell and Schrödinger equations.

On the other hand, the quantum physics is useless in the domain of ordinary electric circuits. It seems a precipice separates theories.

But there is a bridge; the formula of Lorentz force in electrodynamics can be gain as a solution of a quantum equation [10]. Another way can be constructed employing the laws of energy and momentum conservation, unifying the description of the same phenomena using different models (to see below).

\section{EMISSION OF NEUTRAL HYDROGEN ATOM IN RUTHERFORD - BOHR MODEL}

\section{A. The Electromagnetic Field Model}

In order to relate two approaches - electro dynamical and quantum mechanical, and clarify a common ground, the problem of a hypothetic electron fall in the nucleus of hydrogen is considered, and the planetary model of atom is taken. Although Bohr's model is primitive and obsolete, it can lead to the payoff.

Electron at the first Bohr's orbit releases all its energy as it falls down to the ground energy state, i.e. into nucleus, and annihilates. This energy and with it the momentum are emitted into a space in the form of an electromagnetic wave. 
According to the Maxwell's field theory these energy and momentum are determined by the vectors of corresponding flux. On the other hand, the magnitudes of electron energy and momentum, which must be released with transitions between Bohr's orbits, are known from quantum mechanics as the solution of Schrödinger equation [7].

The underpinning supposition is made: the ratio of momentum flux density to the energy flux per unit time is equal to the ratio of the total electron momentum to its energy. This can be expressed by the other way: the fluxes of energy and momentum are proportional to total energy and momentum themselves, what is resulted by integrating the flows over time and space. As is known, fluxes are defined by Poynting vector [7], [11].

Poynting vector represents the directional energy flux density (the energy transfer per unit area per unit time) of an electromagnetic wave and expressed by SI units as:

$$
\mathbf{S}=\varepsilon_{0} c^{2} \mathbf{E} \times \mathbf{B}
$$

where $\varepsilon_{0}$ - electric constant,

$c$ - speed of light.

$\mathbf{E}$ and $\mathbf{B}$ - electric field strength and magnetic induction.

The momentum flux density is given by the avowed vector [7], [12], [13]:

$$
\mathbf{g}=\mathbf{S} / c^{2}
$$

Such expression means, that $\mathbf{g}$ and $\mathbf{S}$ are parallel, though the force, exerting on a charge, is aimed in tangential direction, i.e. perpendicular to the wavefront propagation. It is evident, that $\mathbf{g}$ should be determined differently. Therefore, instead of (2) a hypothetic vector of the momentum flux is taken as [14]:

$$
\mathbf{D}=4 \pi \varepsilon_{0} \frac{\partial \mathbf{E}}{\partial t} \times \mathbf{A}
$$

where A - magnetic vector potential.

The factor $4 \pi$ is put because SI units is employing.

Formula (3) is obtained by means of the notion of imaginary vectors and can be considered as a hypothesis [14]. Usually, complex numbers are introduced in calculations as an artificial way to facilitate a computing, and after a calculus the imaginary part is rejected. For example, while using the phasor in the electric circuit theory [15]. But in the present reasoning imaginary vectors have intern deep nature, they are derived from the concept, that the time is the imaginary value. Hence, after calculation the imaginary part of obtained expressions can remain [14].

According [14], the mathematical procedures under polar and axial imaginary vectors differ and obey some specific rules. An application of them let to obtain the new momentum flux formula (3). Here $\mathbf{D}$ is perpendicular to $\mathbf{S}$ and depends on the frequency of a wave (due to $\partial E / \partial t$ ).

Returning to the present investigation, in order to derive the ratio of fluxes it is necessary to find the directions of vectors $\mathbf{E}, \mathbf{B}, \mathbf{A}$ in Bohr's model. They are depicted in the
Fig. 1 and built from the point of an electron position in the orbit (for more clarity $\mathbf{A}$ is shifted).

The induction $\mathbf{B}$ is perpendicular to the orbit plane $x O y$. This follows from the symmetry - the circling electron is like a ring current. The direction of $\mathbf{B}$ does not change in time, hence the vector $\partial \mathbf{B} / \partial t$ also pointed along $z$ axis. Vector $\mathbf{E}$ lies radially into the plane $x O y$ according the rotational symmetry. Consequently, $\partial \mathbf{E} / \partial t$ is directed the same.

Vector $\mathbf{A}$ is considered to be tangential to the electron orbit because the definition $\mathbf{B}=\nabla \times \mathbf{A}$.

Such, all necessary vectors either are mutually perpendicular or parallel. This gives a possibility to pass easily from the vector equality to the scalar one. It is enough only to change the vectors by their corresponding modules.

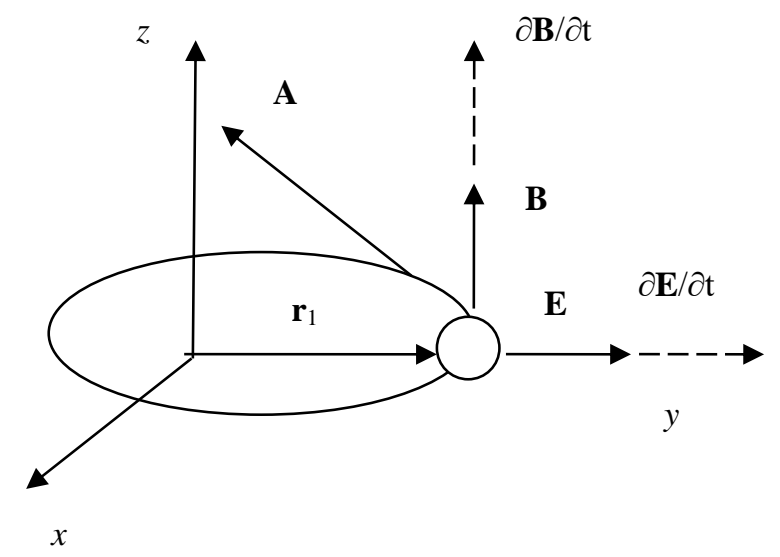

Fig. 1. Electromagnetic field into a hydrogen atom.

In order to calculate the ratio $|\mathbf{D}| /|\mathbf{S}|$ it is needed to know functions $\mathbf{E}, \mathbf{B}, \mathbf{A}$, describing an emission of electromagnetic wave while an electron falls.

The behavior of $\mathbf{E}$ and $\mathbf{B}$ obey the wave equation, that follows from Maxwell's theory with Lorenz gauge condition. Its common solution is $f(r-c t)$, where $r$ - a space coordinate. Because an emission spectrum of hydrogen is linear determined by the Balmer series, then the wave is considered to be sine monochromatic with the angular frequency $\omega$. Hence, considering the first orbit and admitting for brevity $r$ $=0$, next equalities follow:

$$
\begin{aligned}
& E(t)=E \sin \omega_{1} t \\
& \frac{\partial E(t)}{\partial t}=\omega_{1} E \cos \omega_{1} t
\end{aligned}
$$

In order to determine $\mathbf{A}$ it is important to evaluate the variation of $\mathbf{B}$ into an atom, that is unknown. However, from the Stokes theorem it is established that for the circular contour in the homogenous magnetic field next expression holds:

$$
\mathbf{A}=0.5 \mathbf{B} \times \mathbf{r}
$$

If the field is not homogenous, the other factor instead of 0.5 must be put. In case of the first orbit the simplest formula is assumed: 


$$
\mathbf{A}_{1}=\mathbf{B}_{1} \times \mathbf{r}_{\mathbf{1}}
$$

Otherwise, the result will be obtained up to an unknown factor of the order of unity. Because the modules of the sine and cosine are equal (unity), and using (1), (3), (5) and (6), the ratio of fluxes may be expressed as:

$$
\frac{|\mathbf{D}|}{|\mathbf{S}|}=\frac{4 \pi \omega_{1} r_{1}}{c^{2}}
$$

According the main initial considerations there is:

$$
\frac{|\mathbf{D}|}{|\mathbf{S}|}=\frac{\left|\mathbf{p}_{1}\right|}{\left|\mathrm{E}_{1}\right|}
$$

where $p_{1}$ and $\mathrm{E}_{1}-$ momentum and energy of an electron, when it falls into nucleus and annihilates.

The left-hand side in (8) matches the classical physics and the right one - quantum theory. This relationship gives a key for the further investigation.

\section{B. The Quantum Physics Model}

By means of quantum mechanics the momentum module of an electron on the first Bohr's orbit is given by:

$$
p_{1}=\hbar / r_{1}
$$

where $\hbar$ - reduced Planck constant.

The radius $r_{1}$ can be expressed using physical constants as:

$$
r_{1}=\frac{4 \pi \varepsilon_{0} \hbar^{2}}{m e^{2}}
$$

where $e, m$ - charge and mass of an electron.

Substituting (7), (9) and (10) into (8) yields a ratio:

$$
\frac{\hbar}{\mathrm{E}_{1} r_{1}}=\frac{4 \pi \omega_{1} r_{1}}{c^{2}}
$$

After simple rearrangements the formula of energy follows:

$$
\mathrm{E}_{1}=\frac{m^{2} c^{2} e^{4}}{(4 \pi)^{3} \varepsilon_{0}^{2} \hbar^{3} \omega_{1}}
$$

Keeping in mind, that the electron energy $U_{1}$ on the first orbit as the solution of non relativistic Schrödinger equation [16], [21] is equal:

$$
U_{1}=\frac{m e^{4}}{(4 \pi)^{3} \varepsilon_{0}^{2} \hbar^{2}}
$$

then (11) may be rewritten in a kind:

$$
\mathrm{E}_{1}=m c^{2} \frac{m e^{4}}{(4 \pi)^{3} \varepsilon_{0}^{2} \hbar^{2}} \frac{1}{\hbar \omega_{1}}
$$

In the compact form using (12) it takes a view

$$
\mathrm{E}_{1}=m c^{2} \frac{U_{1}}{\hbar \omega_{1}}
$$

Respectively the Planck's theory, energy of the radiation is proportional to its frequency while an electron jump between states (orbits) occurs. So, $U_{1}=\hbar \omega_{1}$ what is just equal to a denominator in (13).

Hence, electron energy of its hypothetic fall and annihilation with proton becomes equal:

$$
\mathrm{E}_{1}=m c^{2}
$$

In a nature such a reaction is not observed, but there is an annihilation of an electron and positron. Thus, $E_{1}$ is the total energy of an electron on the first orbit. An important conclusion follows from (14): the mass of an electron and its energy, which releases by emission, are equivalent. This result is obtained without use of the Relativity.

If an electron falls down to a nucleus from the $n$-orbit, the formulas, describing its states, become as follows [16]:

$$
U_{n}=U_{1} / n^{2}, \quad p_{n}=p_{1} / n, \quad r_{n}=r_{1} n^{2}
$$

Also, it should be admitted a relation $\mathbf{A}_{\mathbf{n}}=\frac{1}{n} \mathbf{B}_{\mathbf{n}} \times \mathbf{r}_{\mathbf{n}}$. The last condition means that an electromagnetic field inside an atom is quantized. This matches a concept of Quantum field theory (QFT) in contemporaneous physics [17].

Putting shown above expressions in $\frac{|\mathbf{D}|}{|\mathbf{S}|}=\frac{\left|\mathbf{p}_{n}\right|}{\left|\mathrm{E}_{n}\right|}$, leads to:

$$
\mathrm{E}_{n}=m c^{2} \frac{U_{n}}{\hbar \omega_{n}}
$$

Because energy of electron transition between $n$ - orbit and a nucleus is accompanied by a radiation and defined as $U_{n}=\hbar \omega_{n}$, then (15) transforms in common result $\mathrm{E}_{n}=m c^{2}=\mathrm{E}$. An interesting conclusion follows: $\mathrm{E}$ remains the same for any orbit, even when $n \rightarrow \infty$, i.e., electron is free. This strange fact can mean that electron annihilation energy depends on the orbit from which electron falls. Perhaps, therefore such phenomena are forbidden, electron never jumps into proton. More probably, an electron should be considered as a wave-like object, its and a field energy are inseparable.:

The formula (8) can be rewritten in the unfolded view:

$$
\frac{\left|4 \pi \frac{\partial \mathbf{E}}{\partial t} \times \mathbf{A}\right|}{\left|c^{2} \mathbf{E} \times \mathbf{B}\right|}=\frac{e^{2}}{4 \pi \varepsilon_{0} \hbar c^{2}}
$$


According to depicted directions of vectors in Fig. 1, (16) transforms into one scalar equality, containing an imaginary unit $i$.

$$
\frac{4 \pi \frac{\partial E}{\partial t} r_{1}}{i c^{2} E}=\frac{e^{2}}{4 \pi \varepsilon_{0} \hbar c^{2}} \quad, \quad i=\sqrt{-1}
$$

All obtained expressions were based on the hypothesis that the formula (3) of momentum flux, which is real number, holds. At the same time Poynting vector occurs imaginary, i.e., $\mathbf{S} \equiv i \mathbf{S}$ that corresponds with Complex Poynting theorem [18]-[20]. Therefore, the imaginary unit $i$ appears in (17).

After substituting (10) in (17) and simple rearrangement this expression turns out be as follows:

$$
\frac{\partial E(t)}{\partial t}=i \frac{m e^{4} E(t)}{(4 \pi)^{3} \varepsilon_{0}^{2} \hbar^{3}}
$$

If an electromagnetic wave is considered in the fixed point of a space, then the partial derivative can be changed by ordinary one, so as:

$$
\frac{d E(t)}{E(t)}=i \frac{U}{\hbar} d t
$$

The solution in the domain of real numbers leads to:

$$
E(t)=E \cos \frac{U}{\hbar} t
$$

where $U$ - energy difference between quantum states, an integrating constant is denoted as $E$ (wave amplitude).

This result coincides with an initial adoption of an emitted wave (4). It seems worth mentioning that the solution is obtained in a kind of a sine, i.e., monochromatic wave with a linear emission spectrum and a frequency $\omega=U / \hbar$.

\section{RESUlT AND DISCUSSION}

The problem of a hypothetic electron fall into a nucleus of hydrogen atom by means of two different concepts - classical electrodynamics and quantum physics, is examined. It was demonstrated that energy of a free electron is equal $m c^{2}$ without any mentioning of the Special Theory of Relativity. This energy and an electron mass are equivalent and transmutable. Such conclusion is succeeded by utilizing Maxwell's field theory and Bohr's atomic model conjointly. The result is obtained using the laws of energy and momentum conservation including a case of electron annihilation. The solving is built on an assumption that fluxes of energy and momentum densities are proportional to magnitudes of electron energy and momentum in the quantum interpretation. It was shown that an electromagnetic field inside an atom is quantized and can be described by discrete series of values $\mathbf{E}_{\mathbf{n}}, \mathbf{A}_{\mathbf{n}}$, corresponding with appropriate Bohr's orbit.
In the model of a hydrogen atom, it was shown that postulates of quantum physics and field theory complete each other. Solutions of Maxwell's equations applying to Bohr's model confirm the fundamental fact that electron can behave either like a point object in the quantum stochastic description or like an electromagnetic wave according to wave - particle duality. Both representations are unified by laws of energy and momentum conservation. In case of quasi static states, the quantum model is applicable, when the states change at time the field theory in which field is quantized holds. As a matter of fact, this idea copes with postulates of contemporaneous physics [9], [17].

Additionally, the exposed approach proves the correctness of the propositioned formula of the momentum flux density (3). It states that the momentum of a wave depends not only on electric and magnetic field, but also on the frequency of emission $\omega$.

\section{CONCLUSION}

The present investigation proves an intrinsic link between classical and quantum physics. An essence of an approach consists in the idea, that energy and momentum characteristics of an electron can be described from different points of view, using either the field or quantum models. Differential equations describe continuous phenomena like electromagnetic wave while quantum processes are discrete. But the ratio of fluxes and discrete magnitudes, having a different nature, can be compared as it was made in the present work. Continuity and discreteness were connected in the same equation (8), which issues from conservation laws. The fluxes are determined by two vectors: the Poynting's one and hypothetic $\mathbf{D}$, which is introduced phenomenologically by means of the concept of imaginary time. Its correctness is proved by the concordance of final results.

The propositioned approach let to obtain the main achievement - energy of a free electron is defined by the famous formula $m c^{2}$ without mentioning of Relativity, and which is deduced only from Maxwell and non relativistic Schrödinger equations.

An interesting consequence follows from (13). The two crucial definitions of quantum energy $m c^{2}$ and $\hbar \omega$ are interrelated. They are derived on the base of Maxwell's theory and Bohr's atom model. If $\hbar \omega$ is adopted according to Planck's equation, then Einstein's formula $m c^{2}$ appears automatically and vice versa.

The exposed approach proves one more, that the description of quantum objects must be completed by gauge field theory. The basic ideas of Quantum electrodynamics are illustrated with use Bohr's atom model, in which field, radiation and matter are mutually converted. Annihilation energy of an electron, as well known, transforms in energy of an electromagnetic emission.

\section{REFERENCES}

[1] Gabriel D. Puccini. "Electrodynamics under a possible alternative to the Lorentz transformation". Foundations of Physics Letters. 16(5), 481-494, Jan. 2017. DOI: 10.1023/BFopl0000012778.72452.6f.

[2] E.T. Kinzer, J. Fukai. "Weber's force and Maxwell's equation". Found. Phys. Lett. 9(5), p.457. 1996. DOI: 10.1007/BF02190049. 
[3] T.K. Sarkar, M. Salazar-Palma. "Maxwell's original presentation of electromagnetic theory and its evolution", in Hand Book of Antenna Technologies. Springer, Singapore. 2015. DOI: org/10.1007/9789814560.

[4] A.L. Shalyapin, V.I. Stukalov. Introduction in classical electrodynamics and atomic physics. Yekaterinburg. UMC. 490p 2006. (in Russian).

[5] V.M. Bernstein. "Evolution of Gauss - Weber electrodynamics, quantum mechanics without a wave theory", in Fundamental problems of natural science and techniques. 30p. Saint Petersburg 2000. (in Russian).

[6] F.F. Mende, A.S. Dubrovin. Alternative ideology of electrodynamics. Monograph, Moscow, 218p. 2016. (in Russian).

[7] R.P. Feynman, R.B. Leigton, M. Sands. The Feynman lectures on physics. Massachusetts, London, vol. 2, 1964.

[8] L-M. Moukala, T. Nsongo. "A Maxwell like theory unifying ordinary fields". Research Journal of Engineering Sciences. vol. 6(2), 20-26, Feb. 2017.

[9] R.P. Feynman. QED - the strange theory of light and matter. Memorial lectures. Alix G. Mautner. Princeton University Press. 1985.

[10] Y.E. Khoroshavtsev. "The speculation on quantum mechanics proceeding from a principle of the conservation of entropy at an atomic scale". Journal of Mathematics Research. ISSN 1916 - 9795. vol. 11(3), 1-16, 2019. DOI: 10.5539/jmr.v11n3pxx.

[11] Francis S. Johnson, Bruce L. Cragin, Richard Hodges. "Electromagnetic momentum density and the Poynting vector in static fields". American Journal of Physics. 1994. DOI: org/101119/1.17738.

[12] S.M. Kim, Greg. Gbur. "Angular momentum conservation in partially coherent wave fields". Phys. Rev. A (86), Oct. 2012.

[13] R. Pfeifer, T. Nieminen, N. Heckenberg. "Momentum of an electromagnetic wave in dielectric medium". Reviews of Modern Physics. 79(4), 2007.

[14] Y.E. Khoroshavtsev. "Imaginary vectors in mechanics and electrodynamics". Bulletin of Civil Engineers. ISSN 1999-5571, 4(9), 115-117, Dec. 2006. (in Russian).

[15] David R. Jackson. The electrical engineering hand book. Edited by: Wai-Kai-Chen, Academic press. 2005.

[16] R.P. Feynman, R.B. Leigton, M. Sands. The Feynman lectures on physics. Addison - Wesley publishing company, Massachusetts, London, Vol. 3, 1963.

[17] Matthew D. Schwards. Quantum field theory and the standard model. University Press, Cambridge, New York, 1st edition. March 2014. DOI: doi.org/10.5281/zenodo.2578864.

[18] T.D. Carozzi, J.E.S. Bergman, R.L. Karlsson. "Complex Poynting theorem as conservation law". Preprint. 2005.

[19] Gerald Kaiser. "Completing the complex Poynting theorem: conservation of reactive energy in reactive time", in Center for Signals and Waves, Portland. Dec. 2014.

[20] Ignacio Campos Jimenez, J.A. E. Roa Neri. "About Poynting's theorem". European Journal of Physics. 13(3), 117, July 2000. DOI: 10.1088/0143-0807/13/3/003.

[21] L.D. Landau, \& E.M. Lifshitz. Quantum mechanics: Non-Relativistic theory. vol. 3, 3-rd, ed. 1977. 